\title{
The Application Conception of OTN\&PTN in the Power Distribution Automation
}

\author{
Bi Qing-gang ${ }^{1}$, Li Yang ${ }^{2}$,Luo Xian-nan ${ }^{3}$ \\ (State Grid Electric Power Research Institute,Nanjing,China,210003) \\ biqinggang@sgepri.sgcc.com.cn; liyang2@sgepri.sgcc.com.cn \\ luoxiannan@sgepri.sgcc.com.cn
}

Keywords:The smart power grids, the distribution network, OTN, PTN

\begin{abstract}
The Optic packet transport is the ideal technology, that realizes seamless connection of the optical transport network and electric layer IP network. The paper studies two kinds of current mainstream technology, include the optical transport network and the packet transport network, and discusses the characteristics, network application position and network construction of the two technologies, Based on the needs of smart power distribution automation telecommunication service over IP, the paper brings forward a way to the application of OTN\&PTN in the power distribution automation.
\end{abstract}

\section{Introduction}

With the development of the smart grids, the application requirement of data, voice and video service is increasing, and business demand produced very big change. So that, these put forward higher requirements for electric power communication, included of real-time, safety, the efficiency of bandwidth, QoS guarantee, network protection and restoration etc, and also adapt to the evolutionary trend of distribution network office system and monitoring system to IP. The appearance of PTN(Packet Transport Network), based on packet transport core and combination of the advantage of traditional transport network and data communication network, right meets with the new requirement of the development of distribution automation communication system ${ }^{[1]}$.

\section{The Requirement Analysis of Distribution Automation Communication Network}

The power network with voltage of $110 \mathrm{kv}$ or less belongs to distribution network( including of high voltage, medium voltage and low voltage),that to be discussed is specific reference to the $10 \mathrm{kv}$ medium voltage distribution network automation. At present, the first batch of pilot cities of State Grid adopt the first architecture. The distribution automation expresses a integrated automation system at first, and can monitor, coordinate and manage each link equipment and optimal operation of the whole distribution network in real-time condition. Distribution automation is a systematic engineering, which includes four main links: power supply network, telecommunication system and master station network. We can see the telecommunication occupies a quite important role in distribution automation, and it undertakes the task that data transmission and order issuance between the distribution network monitoring center and switchgear station equipment (such as RTU, automation alarm device and load control device etc). From the point of view of communication channel, there are mainly four communication modes( including wire communication, wireless communication, optical fiber communication and carrier communication). Figure 1 show the architecture of distribution automation traditional communication system. 


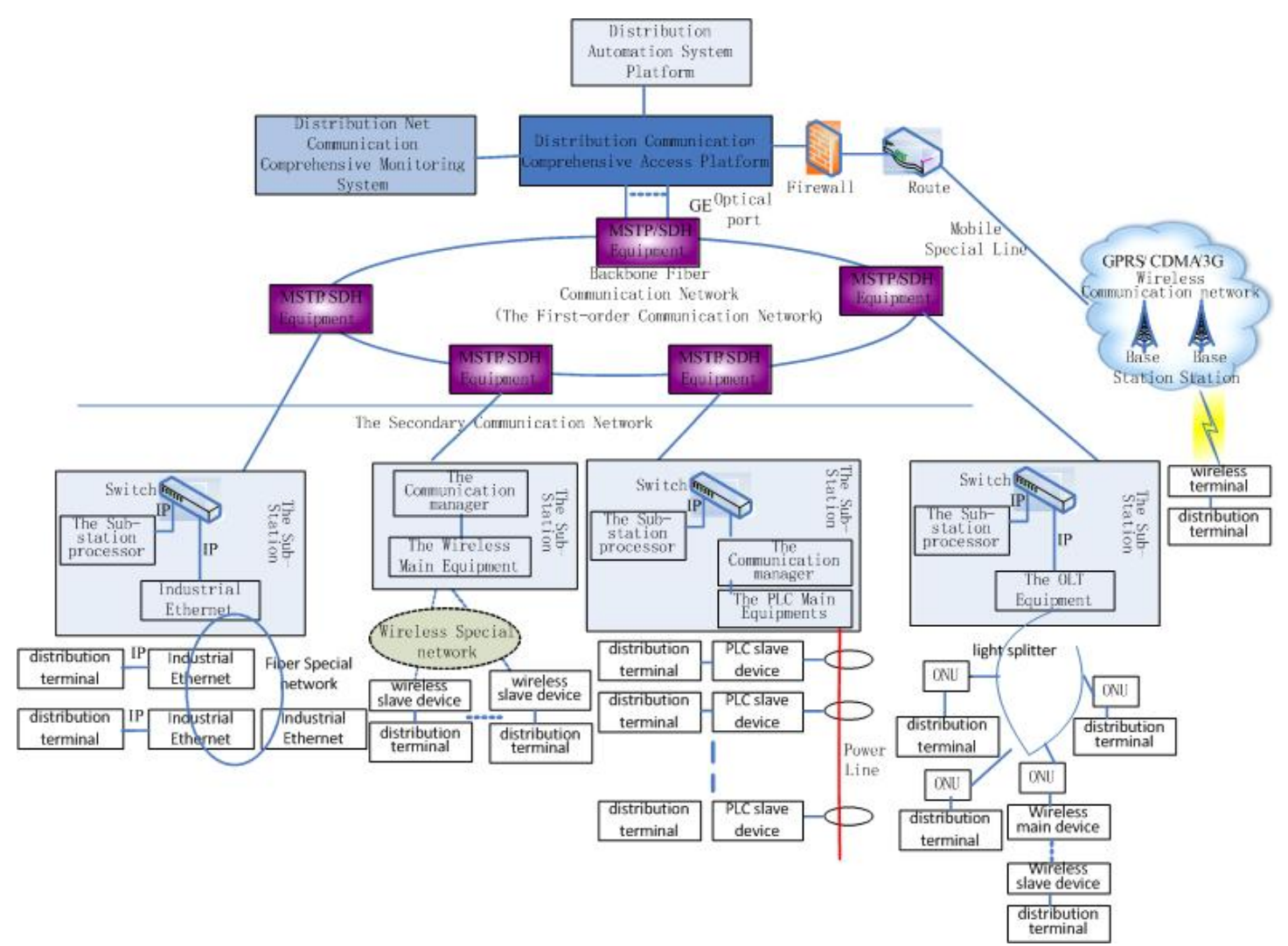

Fig. 1 Architecture graph of distribution automation traditional communication system

With the development of electric power communication, the main mode of communication service is changing from voice communication to data communication. For example, Sichuan Xiangjiaba Dam-Shanghai $\pm 800 \mathrm{kV}$ HVDC Demonstration Project, that is the HVDC project of the highest voltage grade, the farthest transmission distance and the maximal capacity ${ }^{[2]}$. According to the statistics of preliminary design documents in matching fiber communication project, the emphasis of power application is changing from voice communication to data communication. The traffic of data communication has exceeded $80 \%$ of the total bandwidth demand. Moreover, the IP protocol occupies the leading position in all kinds of data service.

At present, distribution communication networking adopts MSTP/SDH. For SDH network, initially we mainly considered the TDM signal based on circuit switching, and only considered the ATM at the beginning for packet signal, but not the data service such as IP. When the data service(IP etc) appeared, SDH technology just adapted it into SDH network. But when the data service(IP etc) becomes dominant service, this solution presented deficiency obviously. The development trend of IP-based distribution telecommunication service make the electric power communication develop to the next generation. The appearance of OTN \& PTN rightly cater to this tendency.

\section{Key Technologies Analysis}

\section{Knowledge of the OTN}

The OTN(Optical Transport Network), that represents development direction of the next generation transport network, integrates the advantage of SDH and DWDM, melts transport and switching to one body, and is a ideal platform that carries broadband IP service ${ }^{[3]}$.

OTN mainly locates in metro network and backbone network, carrying and scheduling of large particles, so it has larger application space in backbone layer and aggregation layer of local transmission network. Figure 2 show the networking scene. But it is likely important to pay attention to the bandwidth assignment of OTN is rigid, and utilization ratio of bandwidth is low. Moreover, it is difficult to process smaller particle service. The PTN just can be make up for defective. 


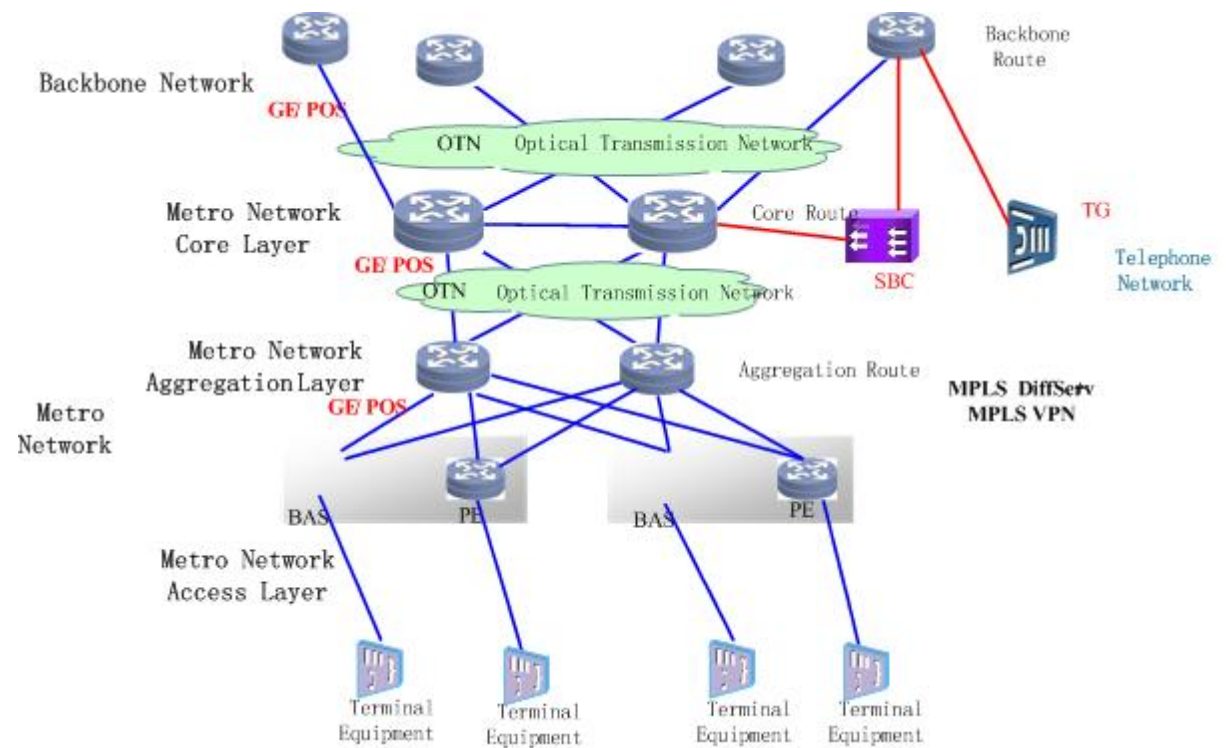

\section{Knowledge of the PTN}

Fig. 2 The OTN Networking schematic diagram

PTN(Packet Transport Network) is the new generation transport network technology, that takes packet exchange as key and orients to packet data service. PTN is a product that combined with SDH, MPLS exchange and Ethernet, but exceeded these technologies. PTN is a intelligent network that orients to future all IP service ${ }^{[4]}$.

The management complexity of PTN equipment is higher than traditional transmission equipment, but smaller than switching capacity. The PTN equipment mainly localized in the bandwidth sensitive access layer and convergence layer. Figure 3 show the PTN networking scene.

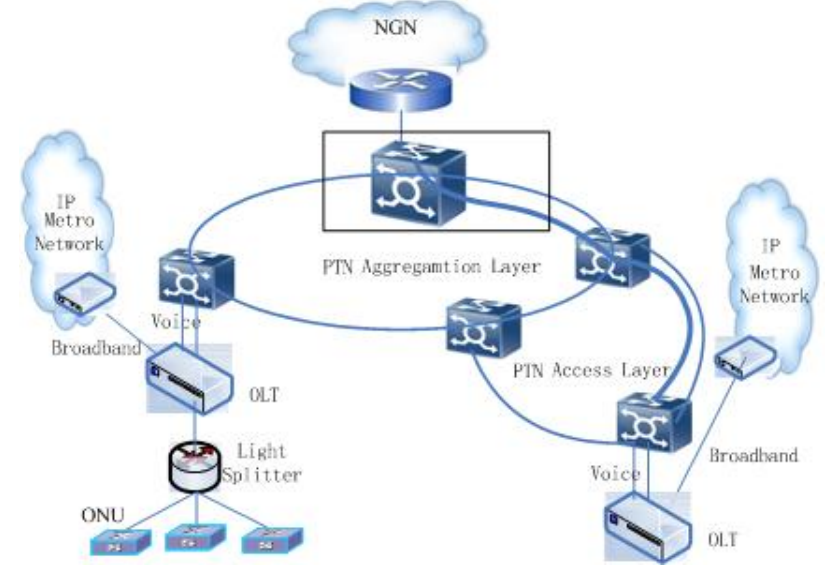

Fig. 3 The PTN Networking schematic diagram

In a word, combined with the technical features of OTN and PTN, we can solve the requirement of large capacity scheduling and fine granularity adjustment very well and realize a good transfer mode for the different service. It has appeared greatly important to adopt OTN\&PTN combined networking mode ${ }^{[5]}$. With resort to its powerful capacity of IP access, assembly and flexible scheduling, OTN\&PTN combined networking mode is beneficial to promote to construct unified and fusion flat metro transmission network.

\section{The Application of OTN\&PTN in the Distribution Network}

OTN used as the large capability networking technology that has Optoelectronic joint operation. Electric layer realizes the scheduling based on wavelet, such as 2.5G, GE, 10GE etc. Optical layer scheduling mainly locates in the backbone/core layer with the wavelength of 10G or 40G, while PTN is similar with the MSTP and apply to convergence layer or access layer usually. In present network, the OTN\&PTN combined networking adapts OTN in core backbone layer usually, and adapts PTN under convergence layer, meanwhile makes full use of OTN dispatching the uplink 
service to landing terminal the PTN service pertained. It is need to notice, on the combined networking mode, the OTN is not only a carrying method, but also dispatching the GE/10GE service backbone node upward jointed and cross landing equipment. The number of uplink GE/10GE channel can be distributed according to the service total number the PTN actually accessed, thus it is greatly to simplify the network set up between the backbone node and core node, avoid all node equipments of loop must upgrade because of one node service upgrading on PTN independent network mode, and save the network investment greatly. The service converges on convergence layer and upward joints to large capacity cross landing equipment of core room through combining the optical port with RNC, where the backbone node PTN equipment through GE optical port only connects with the PTN cross equipment that RNC node owned. The service converge on the convergence layer, uplink to core room large capacity cross landing equipments and connect with RNC through GE port, where The backbone point PTN equipment only connects with the PTN cross equipment which belongs to RNC point, but doesn't relate with the PTN cross equipments of the other RNC nodes and the backbone PTN equipments of the convergence ring.

At present, the distribution network automation technology is not enough mature, so it conforms to reality principle by using mixed communication program.

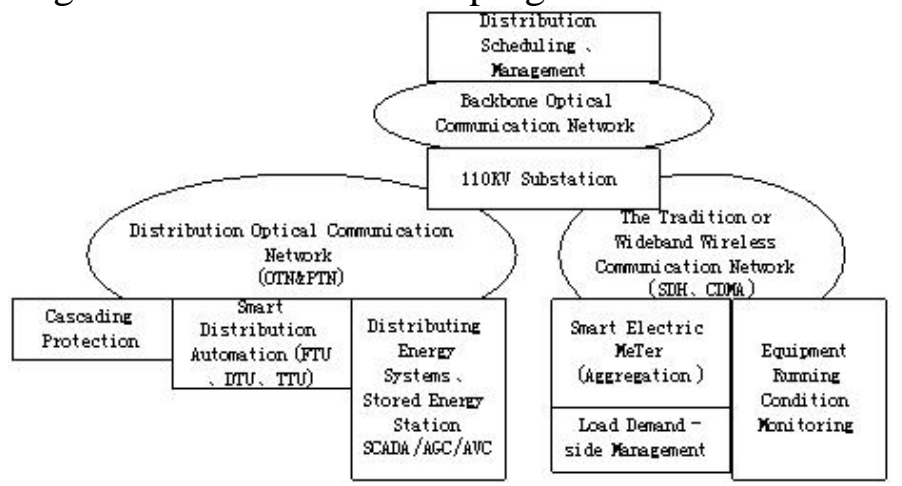

Fig.4 Smart Distribution Grid Communication Network Technology program diagram

\section{Summary}

The OTN\&PTN ,that is a hot networking structure technology ,is introduced demanding for the next generation optical transmission system. Although its application is developing, the prospect is very optimistic because of the designing of OTN\&PTN, that integrates the advantage of the protocols and technology, such as SDH/MSTP,ATM,Ethernet,MPLS and WDM etc. It demands to apply it to distribution communication network of distribution automation adapting to the smart grid. The technology of OTN\&PTN will surely become one of best technologies, that the next optical transmission power communication system networking, owns the characteristics that advantage of the technology, superiority of performance, effectiveness of investment and multiplicity of supports.

\section{References}

[1] Li Xiang-zhen, He Qing-su, Sun Ji-sheng: Research and Application about Intelligent Distribution Network Communication Technology[J].ELECTRIC POWER, 2011,12:78-81.

[2] Preliminary Design of Sichuan Xiangjiaba Dam-Shanghai \pm 800kV HVDC Demonstration Project Matching Fiber Communication Protect[Z]. South Electric Power Design Institute, 2009.

[3] Zhao Wen-yu, Zhang Hai-yi, Tang Rui, Wu Qing-wei: Key Technology and Application Strategy about OTN[J], Telecommunications Network Technology, 2010,11:50-54.

[4] Gong Qian, Xu Rong, Li Yun-bo: Packet Transmission Network[M]. POSTS \& TELECOM PRESS, BeiJing,2009.

[5] Wei Tao, Zhang Bin: Combined Network Model Analysis of OTN+PTN[J]. Telecommunications Science. 2010 26(7):132-136. 